\title{
Bjomédica
}

Articulo Original

\section{Portadores faríngeos de Streptoccocus pneumoniae en dos grupos poblacionales. Ciudad. Bolivar, Estado Bolívar, Venezuela}

Requena-Certad, Ixora ${ }^{1}$, Pacheco-Urbina, Carla ${ }^{2}$, Orta, María de los Angeles ${ }^{1}$, MarcanoGallo, N. ${ }^{1}$, Salazar-González, R ${ }^{1}$.

${ }^{1}$ Calle José Méndez. Edif. Ciencias de la Salud "Francisco Battistini Casalta". Departamento de Parasitología y Microbiología. Ciudad Bolívar, estado Bolívar, Venezuela.

\section{Autor para correspondencia}

Nombre: Requena-Certad, Ixora

Institución: Ciencias de la Salud "Francisco Battistini Casalta".

Departamento: Departamento de Parasitología y Microbiología

Dirección: ${ }^{1}$ Calle José Méndez. Ciudad Bolívar, estado Bolívar, Venezuela.

E-mail: ixorarequena@gmail.com

Recibido: el 07 de septiembre de 2017 Aceptado para publicación: el 12 de diciembre de 2017

Copyright () 2016 por autores (s) y Revista Biomédica.

Este trabajo esta licenciado bajo las atribuciones de la Creative Commons (CC BY).

http://creativecommons.org/licenses/by/4.0/

(c) (i) Open Access 
Requena-Certad et al.

Portadores faríngeos de Streptoccocus pneumoniae en Venezuela

\section{Resumen}

Introducción. Streptococcus pneumoniae es un patógeno para el ser humano que precisa de una previa colonización faríngea para causar enfermedad, cuya morbimortalidad se acentúa en los menores de 6 años y los mayores de 65 años de edad.

Objetivo. Determinar la prevalencia de portadores faríngeos de Streptococcus pneumoniae en dos grupos poblacionales en Ciudad Bolívar, Estado Bolívar.

Metodología. Durante los años 2009 a 2010, se evaluaron dos grupos: uno que incluyó a 66 individuos de la tercera edad institucionalizados en el Asilo "San Vicente de Paúl" y Geriátrico "Carlos Fragachan", con una edad promedio de 75 años \pm 6 años y otro que abarcó a preescolares y escolares de 2 a 12 años hospitalizados en los Servicios de Pediatría y del área de emergencia del Complejo Hospitalario Universitario "Ruíz y Páez”. A cada individuo se le tomó una muestra faríngea, la cual fue procesada según los lineamientos establecidos por la Sociedad Americana de Microbiología. Se estudiaron 80 exudados de origen faríngeo. Resultados. En el grupo de los individuos de la tercera edad se identificaron 10 casos $(15,15 \%)$ de portadores faríngeos de $S$. pneumoniae. El $70 \%(\mathrm{n}=7)$ correspondía al género masculino. En todas las edades se diagnosticaron casos, frecuentemente observados en el grupo entre 71 a 80 años de edad $(n=4 ; 40 \%)$. El 46,2\% de los portadores faríngeos de la tercera edad refirieron antecedentes tabáquicos. Se observaron diferencias estadísticas significativas entre el estado de portador de neumococo y la diabetes mellitus tipo II y estado de vacunación contra la bacteria, En el grupo de los pacientes preescolares y escolares hospitalizados, se aislaron 3 cepas de S. pneumoniae que representó el 3,75\% del total. El mayor porcentaje de muestras positivas se obtuvo en el grupo de 2 a 4 años, con predominio del género masculino (2,5\%). En el grupo de la población infantil hospitalizada, se observaron diferencias estadísticamente significativas entre el estado de portador y antecedentes personales como asma, resfriado común a repetición, infección urinaria e infección de piel y tejido blando. No estaban vacunados contra el neumococo o solo cumplieron una sola dosis. En ambas investigaciones se determinaron 13 cepas de Streptococcus pneumoniae, las cuales mostraron un perfil de resistencia a la Penicilina, por método del disco de Oxacilina, del 50\%; mientras que la totalidad de las cepas aisladas resultaron con alta resistencia a Macrólidos, Clindamicina y Sulfamidas, y sensibles a Vancomicina.

Conclusión: Se identificó una baja prevalencia de individuos colonizados por Streptococcus pneumoniae, sin embargo, se debe considerar la investigación de colonización faríngea por neumococo como un buen método por ser simple y fácil de obtener muestras bacterianas y poder reflejar la progresión de la resistencia bacteriana en grupos de riesgo. 
Palabras Clave: Streptococcus pneumoniae, tercera edad, pacientes pediátricos, resistencia antimicrobiana

\section{Abstract}

Introduction. Streptococcus pneumoniae is a pathogen for humans that requires a prior pharyngeal colonization to cause disease, whose mortality is accentuated in children 6 years and older than 65 years of age.

Objective. To assess the prevalence of pharyngeal carriage of $S$ pneumoniae in two population groups in Ciudad Bolívar, Bolívar State.

Methodology. During the years 2009 and 2010, two groups were evaluated: one which included 66 senior individuals institutionalized in the asylum "San Vicente de Paúl" and the geriatric "Carlos Fragachan", with an average of 75 years \pm 6 years age and another that it comprised preschool and school aged 2 to 12 hospitalized in Pediatrics and the area of the University Hospital emergency services "Ruiz and Páez". Each individual took a pharyngeal sample, which was processed according to the guidelines established by the American society for of microbiology. 80 exudates from pharyngeal origin were studied.

Results. Between elderly individuals 10 cases were identified (15.15\%) of S. pneumoniae pharyngeal carriers. $70 \%(\mathrm{n}=7)$ corresponded to the male gender. In all the ages were diagnosed cases, often observed in the group between 71 to 80 years of age $(n=4 ; 40 \%)$. $46.2 \%$ of pharyngeal carriers concerned smoking history. Were observed significant statistical differences between the pneumococcus`s state carrier and type II diabetes mellitus and vaccination's state against the bacterium, In the group of preschool and school inpatients, 3 strains of $S$. pneumoniae that accounted for $3.75 \%$ of the total were isolated. The highest percentage of positive specimens was obtained in the group of 2 to 4 years, with a predominance of the male gender $(2.5 \%)$. Statistically significant differences were observed in the hospitalized children, between the carrier's state and personal history as asthma, cold common, urinary tract infection and infection of skin and tissue soft. They were not vaccinated against the pneumococcus or met only a single dose. Both studies identified 13 strains of Streptococcus pneumoniae, which showed a profile of resistance to penicillin, by method of disk Oxacillin, of 50\%; While all of the isolates were with high resistance to macrolides and clindamycin, sulfonamides, and sensitive to Vancomycin.

Conclusion: We identified a low prevalence of individuals colonized by Streptococcus pneumoniae, however should be consider the research of colonization of the pharynx by Pneumococcus as a good method to be simple and easy to obtain bacterial samples and to reflect the progression of bacterial resistance in risk groups.

Keywords: Streptococcus pneumoniae, elderly, pediatric patients, antimicrobial resistance 
Requena-Certad et al.

Introducción

Streptococcus pneumoniae (conocido como neumococo) es un comensal frecuente del epitelio respiratorio de niños y adultos aparentemente sanos ${ }^{1}$. S. pneumoniae es el principal agente bacteriano causante de infecciones del tracto respiratorio, incluyendo otitis, sinusitis, neumonías y reagudizaciones de bronquitis crónica ${ }^{2}$. Sin embargo, el neumococo es una causa importante de enfermedades invasivas e infecciones del tracto respiratorio y causa aproximadamente 500.000 muertes en los niños menores de 5 años de edad sobre todo en países de bajos ingresos ${ }^{3-4}$.

En Venezuela, para el año 2013, se registró una tasa de mortalidad por causas infecciosas, según edad (100.000 hab) del 66,3. Las principales causas de muerte por bacterias fueron producidas por Staphylococcus aureus, Streptococcus pneumoniae, Pseudomonas aeruginosa, entre otras ${ }^{5}$. En la actualidad, el mayor problema con el neumococo es la aparición de resistencias a los agentes antimicrobianos ${ }^{6}$ y su elevada morbimortalidad, ya que, a pesar del uso de antibióticos apropiados y de un tratamiento médico correcto, la mortalidad global por las infecciones invasivas se ubica en un 10\%, siendo del 30-40\% en población de edad avanzada ${ }^{7}$. En pacientes ingresados en una unidad de cuidados intensivos la mortalidad es del $36 \%$, y del $50 \%$ en mayores de 64 años ${ }^{8}$. Aunque, como se indica, las tasas más altas se dan entre los lactantes, niños menores de 2 años y personas mayores de 65 años $^{8-9}$, la presencia de ciertas condiciones médicas subyacentes aumenta el riesgo de enfermedad neumocócica invasiva (ENI) y de sus complicaciones, destacando, desde el punto de vista neumológico, el tabaquismo y las enfermedades respiratorias crónicas y el estado de portador nasofaríngeo de S. pneumoniae $e^{10-11}$.

La colonización por neumococo se inicia en la etapa de lactancia. Estudios realizados con seguimiento longitudinal demuestran que los niños son colonizados por S. pneumoniae al menos una vez durante la infancia ${ }^{1,12-13}$; luego se comienza a elevar por encima de los 50 años $^{14}$. La duración del estado de portador es serotipo-dependiente. Además, la producción local de anticuerpos puede ser importante en la limitación de la duración del estado de portador. En adultos mayores, preescolares y escolares, la producción de anticuerpos IgG específicos se desarrollan después de una colonización, y a veces sin evidencia de una infección previa ${ }^{1,9}$. Lo que sí está demostrado es la relación entre el portador asintomático faríngeo en la transmisión de $S$. pneumoniae, la cual generalmente precede a la ENI ${ }^{15}$. 
Los factores de riesgo que favorecen el estado de portador sano de neumococo son: a) El hacinamiento; estancia en centros de cuidado diario, orfanatos o geriátricos, sobre todo porque en ellos se promueven la diseminación y clonación de cepas multirresistentes, b) infecciones virales frecuentes y c) Uso extendido y excesivo de antibióticos, que promueve el reemplazo de cepas susceptibles a los agentes antimicrobianos por nuevas cepas ahora resistentes, d) enfermedades respiratorias de base, e) hábitos tabáquicos ${ }^{14}$.

Pese a todo, aún no está claro si la aparición de la enfermedad en los infantes y los individuos de la tercera edad, posterior a la colonización faríngea de Streptococcus pneumoniae se deba a la virulencia de los distintos serotipos de neumococos o a factores biológicos propios de esos grupos de edad, de allí la importancia de mantener inmunizada a estos grupos de riesgo ${ }^{10}$.

Se conoce una prevalencia, de moderada a alta, entre niños portadores faríngeos de $S$. pneumoniae en países en vías de desarrollo. La tasa varía entre un 35-45\% aproximadamente ${ }^{13}$. Se han descrito tasas de portadores pediátricos o geriátricos de neumococos, superiores al 45\%, en otros países incluyendo Bangladesh ${ }^{16}$, Gambia ${ }^{17}$, Kenia ${ }^{18-}$ 19, Tanzania ${ }^{20}, \mathrm{Gaza}^{21}, \mathrm{Ghana}^{22}$, India $^{23}$, Indonesia ${ }^{24}$ y Vietnam ${ }^{25}$.

Son pocos los estudios publicados con relación a la frecuencia del neumococo en portadores faríngeos en los individuos de la tercera edad, y la distribución de los patrones de resistencia antimicrobiana. En $2007^{26}$ se identificó un 26,3\% $(n=118)$ de individuos de la tercera edad colonizados en el período en estudio. En otra investigación se demostró un estado de portador del 10,3\% en sujetos de 40-49 años ${ }^{27}$. Esta cifra quizás sea explicada por el mayor hacinamiento y mayor contaminación intradomiciliaria.

A pesar de ello, en Venezuela, son pocos los registros conocidos sobre la frecuencia del neumococo en portadores faríngeos en los niños o individuos de la tercera edad, ni la distribución de los patrones de resistencia antimicrobiana ${ }^{28}$. En edad preescolar y escolar se ha determinado porcentajes de colonización que oscilan entre el 12 al 24\% $28-29$. En individuos de la tercera edad, no existe ninguna investigación al respecto. De allí parte la importancia de investigar la condición de portador orofaríngeo en ambos grupos de edad.

\section{Metodología}

Esta investigación fue de tipo descriptivo y transversal. Se realizó en el Laboratorio de Diagnóstico Bacteriológico “Sócrates Medina”, adscrito al Departamento de Parasitología 
Requena-Certad et al.

y Microbiología de la Escuela de Ciencias de la Salud "Francisco Battistini Casalta", de la Universidad de Oriente, Ciudad Bolívar, estado Bolívar, Venezuela, entre Enero del 2009 hasta Diciembre de 2011.

Se evaluaron un grupo de individuos de la tercera edad institucionalizados en el Asilo "San Vicente de Paúl” y Geriátrico "Carlos Fragachán”, en Ciudad Bolívar, estado Bolívar, 2009-2010 y otro grupo de pacientes pediátricos hospitalizados en el Complejo Universitario Hospitalario "Ruíz y Páez”. Ciudad Bolívar. Estado Bolívar, durante el año 2011.

Se solicitó por escrito una autorización a los Directores de los centros geriátricos ya mencionados en la parte anterior, a los padres y/o representantes de los niños donde se explicó los objetivos y alcance de la investigación. A cada individuo de la tercera edad, padres y/o representantes se le solicitó por escrito, su consentimiento para participar en la investigación.

Para la búsqueda de Streptococcus pneumoniae se tomó una muestra de exudado faríngeo, con hisopo estéril, el cual se introdujo en medio de transporte Amies con carbón $\operatorname{activado}^{30}$.

A cada muestra clínica se le aplicó las técnicas convencionales de procesamiento como inoculación en Caldo Todd Hewitt (BBL® Becton Dickinson), suplementado con ácido nalidíxico $(15 \mathrm{ug} / \mathrm{ml})$ y gentamicina $(8 \mathrm{ug} / \mathrm{ml})$, e incubadas por 24 horas a $35^{\circ} \mathrm{C} . \mathrm{Al}$ día siguiente, se tomó una gota del caldo y se diseminó, sobre la superficie de las placas de agar sangre, con el asa bacteriológica utilizando la técnica de siembra por agotamiento. Los medios de cultivos se incubaron en microaerofilia, a $35^{\circ} \mathrm{C}$ durante $24-48$ horas. Transcurrido este lapso de tiempo, se procedió a observar las características del crecimiento bacteriano ${ }^{31}$; se realizaron pruebas bioquímicas y de susceptibilidad, siguiendo los lineamientos de la $\mathrm{CLSI}^{32}$.

Se definió como portador de $S$. pneumoniae a aquel individuo que no padeciera un cuadro respiratorio agudo en el momento del estudio y del que se hubiera obtenido una muestra faríngea con crecimiento de S. pneumoniae. Se excluyeron del estudio aquellos que, por diferentes motivos, estuvieran tomando tratamiento antibiótico el día de la obtención de muestra clínica. Se definió como cuadro respiratorio agudo la aparición brusca de al menos dos síntomas de los siguientes: tos, expectoración, disnea y dolor torácico, o bien aparición brusca de cuadro febril y al menos uno de los síntomas citados. 
Recoleccion de los datos

Se diseñó un instrumento de recolección de datos, en el cual se vaciaron los datos clínicos, epidemiológicos y bacteriológicos de cada individuo evaluado:

Identificación del paciente, sexo, edad, dirección, servicio donde estaba hospitalizado o si provenía de la consulta externa (en el caso de los preescolares y escolares), tiempo de estancia en la institución geriátrica, antecedentes personales, estado vacunal, resultados del cultivo bacteriano. Además, se anotaron los resultados de la susceptibilidad antimicrobiana de las cepas aisladas, clasificada en sensible, sensibilidad intermedia o resistente, según lo interpretado en las tablas del $\mathrm{CLSI}^{32}$. Se valoró la susceptibilidad antimicrobiana de la bacteria frente a oxacilina, si se observó un halo de inhibición $\geq 20 \mathrm{~mm}$, se consideró como una cepa susceptible penicilina, ampicilina, amoxicilina/ácido clavulánico, ampicilina/sulbactam, cefalosporinas de $1^{\circ}, 2^{\circ}$ y $3^{\circ}$ generación, imipenem y loracarbef. Esto equivale a una $\mathrm{CIM} \leq 0,06 \mu \mathrm{g} / \mathrm{ml}$.

-Aspectos bioéticos

Además del consentimiento informado, se entregó a cada individuo participante, el resultado de su evaluación. Este trabajo se realizó apegado a las normas de investigación en seres humanos según la Declaración de Helsinki ${ }^{33}$.

- Análisis estadístico.

La presentación de los datos se realizó mediante la distribución en cuadros simples según las variables señaladas en la recolección de datos de la investigación. Los datos fueron analizados mediante el programa SPSS para Windows versión 11.0. Los datos de la susceptibilidad bacteriana fueron analizados utilizando el Software Whonet, versión 5 para Windows ${ }^{34}$. Para la comparación de los resultados se aplicó la prueba de Ji-Cuadrado, con un margen de seguridad de $95 \%$. 
Requena-Certad et al.

Resultados

Para el período de estudio se evaluaron 66 individuos de la tercera edad institucionalizados con edades comprendidas entre 51 y 94 años, siendo la media de 75 años \pm 6 años. La mayoría (30,30\%) pertenecía al grupo de 71-80 años. El 53,03\% (n=35) eran del género masculino. Con relación a los pacientes pediátricos hospitalizados se evaluaron un total de 80 pacientes, con edades comprendidas entre 2 a 14 años, siendo la media de 5 años \pm 3 años, de ambos géneros.

En ambos grupos se identificaron 13 cepas de Streptococcus pneumoniae de igual número de portadores asintomáticos. Al discriminarlos por grupos se identificaron tres portadores de $S$. pneumoniae, en el grupo de los pacientes pediátricos hospitalizados, lo cual representó el 3,75 \% (3/80) de la muestra. Dos portadores fueron observados entre los 2 a 4 años de edad (2,50\%), mientras que en el de 5 a 9 años se observó un sólo caso $(1,25 \%)$. Cuando se valoró la relación sexo/portador faríngeo, se determinaron dos pacientes del sexo masculino (2,5\%), y uno de sexo femenino (1,25\%) (Cuadro 1). Por otra parte, en el grupo de los individuos de la tercera edad institucionalizados se aislaron 10 cepas $(15,15 \%), 70 \%$ $(n=7 / 66)$ de los portadores pertenecían al género masculino. Los casos más frecuentemente observados fueron en el entre los 71 a 80 años de edad $(n=4 ; 40 \%)$ para ambos sexos y seguido por el grupo etario (81-90 años) con tres casos (30\%) (Cuadro 2).

En el grupo de la población infantil hospitalizada, se observaron diferencias estadísticamente significativas entre el estado de portador y antecedentes personales como asma, resfriado común a repetición, infección urinaria e infección de piel y tejido blando $(p<00,5)$ (Cuadro 3). Los portadores no estaban vacunados contra el neumococo o tenían inmunización incompleta.

Siete de los portadores faríngeos de la tercera edad (46,2\%) refirieron antecedentes tabáquicos. Se observaron diferencias estadísticas significativas entre el estado de portador de neumococo y la diabetes mellitus tipo II y estado de vacunación contra la bacteria (Cuadro 4).

Las 13 cepas de neumococo mostraron diferentes perfiles de susceptibilidad, con una buena sensibilidad a la oxacilina, como predictor de la sensibilidad a penicilina (15\%), y a las quinolonas $(10 \%)$. Sin embargo, las cepas evaluadas fueron resistentes a eritromicina 
$(64,7 \%)$, levofloxacina (15\%) clindamicina $(100 \%)$ y trimetoprin-sulfametoxazol (100\%). Todas las cepas fueron sensibles a la vancomicina.

\section{Discusión}

La infección invasora producida por Streptococcus pneumoniae aún se asocia a una elevada morbilidad y mortalidad ${ }^{35}$. A pesar del progreso médico, la mortalidad por la neumonía neumocóccica y bacteriémica, sobre todo en adultos, puede ocurrir entre un 6 y $40 \%$, cifra que varía dependiendo de la técnica diagnóstica empleada y la población evaluada $^{36}$. Por otra parte, el aumento de la resistencia de $S$. pneumoniae frente a antimicrobianos de primera línea, como penicilinas y macrólidos, ha derivado en la recomendación del uso de cefalosporinas de tercera generación para el tratamiento de infecciones graves producidas por este microorganismo ${ }^{37-38}$.

En diferentes estudios realizados, se demuestra que la colonización en adultos es bastante menor que la de la población preescolar y escolar. Sin embargo, en esta investigación se encontró una cifra mayor entre los individuos de la tercera edad, quizás explicado por la condición de institucionalizados de los adultos mayores evaluados. Al comparar las cifras de prevalencia de portador faríngeo en la población infantil con la de otros descrita en Venezuela, se observa que son inferiores a otras investigaciones. Se han descrito prevalencias de $12 \%$ en Maracaibo $^{29} ; 13,5 \%$ en otro estudio de la misma ciudad, con una muestra igualmente de 200 preescolares $^{39}$; y 24\% de portadores en Mérida ${ }^{28}$. PerozoMena et $a l^{40}$ refirieron una cifra de portador de neumococo de un $2 \%$.

La situación en Latinoamérica es similar. En México se halló una prevalencia de portadores asintomáticos de $21,4 \%$ de una muestra de 573 lactantes y escolares ${ }^{41}$. En Nicaragua la prevalencia en distintos centros de desarrollo infantil, donde se tomaron muestras de 229 niños menores de 6 años, obtuvieron un porcentaje de colonización que osciló de 23,3\% a 26,6\%, similar al de Venezuela y México ${ }^{42}$. En ambas investigaciones no se excluyeron niños con síntomas respiratorios como en este estudio; además la población analizada no se encontraba en el medio hospitalario como el Hospital Universitario Ruíz y Páez.

La distribución de portadores asintomáticos por S. pneumoniae en cuanto al género no sigue un patrón específico. Según los individuos estudiados, las condiciones clínicas y estado vacunal, la prevalencia de portadores faríngeos de neumococo puede ser mayor entre 
Requena-Certad et al.

los individuos del género femenino ${ }^{14} \mathrm{o}$ del masculino, como se determinó esta investigación. Otros autores ${ }^{29}$ refieren que no existe predilección entre el estado de portador nasofaríngeo de neumococo con el sexo, con lo cual coincidimos.

Los 7 adultos mayores portadores faríngeos de neumococo tenían antecedentes tabáquicos. Se ha demostrado que este hábito afecta el aclaramiento mucociliar y el epitelio respiratorio, y aumenta la adherencia de las bacterias ${ }^{43-44}$. Además, en algunos estudios, los fumadores tienen un nivel de inmunoglobulinas séricas un 10-20\% más bajo ${ }^{45}$ Paradójicamente, los fumadores, parece que tienen niveles aumentados de anticuerpos antineumocócicos, probablemente por un incremento de la frecuencia de infecciones respiratorias ${ }^{46}$. Algunos autores describen mayor frecuencia de colonizaciones nasofaríngeas por neumococo en fumadores, tanto activos como pasivos ${ }^{47}$.

A casi treinta y cinco años después de la primera autorización para aplicar la vacuna de polisacáridos capsulares frente al neumococo, todavía persiste la incertidumbre sobre su eficacia y algunos autores advierten que es preciso revisar, a la luz de los conocimientos actuales, las recomendaciones a favor de su uso sistemático en las personas de 65 años o más ${ }^{49}$. Existe una importante disparidad en las recomendaciones internacionales para su uso en este grupo de edad ${ }^{50}$. Esta polémica se originó a principios de los años ochenta ante la dificultad para demostrar la efectividad de la vacuna en ancianos luego de varios ensayos clínicos $^{51}$. En la actualidad se han realizado siete estudios de metaanálisis que, aunque no han sido concluyentes acerca de la efectividad de la aplicación de la vacuna, no quiere decir que deba considerarse que la misma sea ineficaz, pues estudios observacionales demuestran su beneficio en la prevención de la bacteriemia neumocócica en el anciano y en adultos de alto riesgo ${ }^{52}$.

En este estudio, sólo el $40 \%$ de los portadores estaba vacunado. Este resultado es de relevancia sobre todo en el grupo de los individuos de la tercera edad, pues existen investigaciones que demuestran que quienes han sido inmunizados con la vacuna conjugada heptavalente (aprobada por la FDA en febrero de 2000 e incluida en el calendario en enero de 2001) han sufrido un descenso en la tasa de enfermedad neumocócica (18\% en los mayores de 65 años de edad). Igualmente se ha referido que la aplicación de la vacunación antineumocócica en este grupo de edad proporciona probablemente un cierto grado de 
inmunidad de grupo, por disminución de la tasa de portadores nasofaríngeos, que podría reducir el riesgo de transmisión ${ }^{53}$.

Otro beneficio adicional de la aplicación de la vacuna sería la reducción de los ingresos hospitalarios y las muertes en mayores de 65 años vacunados frente a la gripe y a la enfermedad neumocócica, sobre todo en aquellos con enfermedades cardiopulmonares crónicas $^{54}$.

Las 13 cepas de neumococo mostraron diferentes perfiles de susceptibilidad, con una buena sensibilidad a la oxacilina, como predictor de la sensibilidad a penicilina (15\%), y a las quinolonas (10\%). Sudamérica es una de las regiones más perjudicadas por la resistencia a la penicilina, según los datos de la vigilancia epidemiológica del grupo SIREVA coordinado por la Organización Panamericana de la Salud. Por otra parte, esta resistencia influye marcadamente en el tratamiento de las infecciones invasivas y del sistema nervioso central (meningitis) ${ }^{55}$ pero no las respiratorias. Quizás esto explique porque las cepas aisladas en esta investigación no mostraron una sensibilidad disminuida a la penicilina. En Venezuela, el perfil de sensibilidad del S. pneumoniae a la penicilina en ha variado con los años ${ }^{56,57,58}$.

Esta situación cada día más creciente, difiere a la hallada en el Estado Bolívar, donde un estudio reporta la sensibilidad del $S$. pneumoniae a la penicilina en el Hospital Universitario Ruiz y Páez, estimando que 100\% de las muestras obtenidas eran sensibles a la penicilina. El 41,3\% de los aislamientos presentó sensibilidad disminuida a Oxacilina por método de infusión de discos; sin embargo, al evaluar mediante el método de concentración inhibitoria mínima, no se detectó ninguna cepa resistente a penicilina. Según lo dicho, es probable que las cepas aisladas en el presente estudio sean en su totalidad sensibles a Penicilina $^{59}$.

Por otra parte, las cepas evaluadas fueron resistentes a eritromicina, levofloxacina, clindamicina, y trimetoprin-sulfametoxazol (TXS). A nivel mundial y nacional, el patrón de resistencia a los macrólidos es cada vez mayor. Diferentes publicaciones demuestran que, en promedio, $30 \%$ de las cepas estudiadas mostraban resistencia a la Eritromicina ${ }^{28-41}$ y un $10 \%$ para levofloxacina lo cual es motivo de preocupación ya que éste grupo de fármacos constituyen los antibióticos de primera elección en pacientes alérgicos a betalactámicos ${ }^{42}$, lo cual coincide con los datos establecidos por el Programa Venezolano de Vigilancia de Resistencia Bacteriana a los Antimicrobianos en Venezuela (PROVENRA). 
Requena-Certad et al.

El perfil de resistencia mostrado para clindamicina fue superior al descrito por PROVENRA $^{60}$, quienes ubican dicho porcentaje en un 43,8\% para el año 2001. En el caso de TXS, las cifras a nivel nacional se ubican en un 76,6\%. Casellas ${ }^{61}$ indicó que la resistencia cruzada entre penicilina y TXS, tetraciclina, cloranfenicol y eritromicina y la resistencia a los macrólidos observada en muchos clones es notable. Estos hallazgos, aunque poco determinantes son un ejemplo de la creciente habilidad del Streptococcus pneumoniae para desarrollar resistencia incluso a antibióticos de alta efectividad, limitando muchas veces la terapéutica a un solo fármaco al cual todavía existe una amplia sensibilidad: Vancomicina.

Esta sensibilidad al fármaco mencionado es congruente con los resultados obtenidos en Maracaibo ${ }^{19}$, Mérida ${ }^{18}$ y en otros países de Latinoamérica como México ${ }^{33}$ y Nicaragua ${ }^{34}$. Sin embargo, en el estado Bolívar desde el 2004 se informa 66,7\% de cepas con sensibilidad disminuida $^{55}$. Este fenómeno se debe probablemente a la tolerancia a la Vancomicina descrita en neumococos desde 1999, con una frecuencia estimada del 3 al $8 \%$ de aislamientos tolerantes. Hasta la fecha, no hay reportes de cepas resistentes a vancomicina, sin embargo, la tolerancia es un paso previo para el surgimiento de cepas resistentes en el futuro ${ }^{58}$.

Todas las cepas fueron sensibles a la vancomicina. Este resultado es similar con los obtenidos en Maracaibo ${ }^{29}$, Mérida ${ }^{28}$ y en otros países de Latinoamérica como México ${ }^{41}$ y Nicaragua $^{42}$. Sin embargo, en el estado Bolívar desde el 2004 se informa un 66,7\% de cepas con sensibilidad disminuida ${ }^{59}$. Este fenómeno se debe probablemente a la tolerancia a la Vancomicina descrita en neumococos desde 1999, con una frecuencia estimada del 3 al $8 \%$ de aislamientos tolerantes. Hasta la fecha, no hay reportes de cepas resistentes a vancomicina, sin embargo, la tolerancia es un paso previo para el surgimiento de cepas resistentes en el futuro ${ }^{62}$.

Es importante acotar que sólo una de las cepas mostró un amplio espectro de resistencia a los antibióticos en general. Esta cepa se observó en un niño con antecedente de infección de piel y partes blandas. Quizás haya influido la severidad del cuadro infeccioso, uso extendido y excesivo de antibióticos, promoviendo el reemplazo de cepas susceptibles a nuevas cepas más resistentes ${ }^{63}$. La automedicación con antibióticos contribuye a empeorar este escenario; es importante señalar que muchos representantes manifestaron darles medicación antibiótica a sus hijos, tratándose inclusive de infecciones virales. 


\section{Referencias}

1. Gray B, Converse G, Dillon H. Epidemiologic studies of Streptococcus pneumoniae in infants: adquisition, carriage, and infection during the first 24 months of live. J Infect Dis. 1980 Jun; (142): 923-33.

2. Oteo J, Alós J, Gómez J. Sensibilidad in vitro actual de los patógenos respiratorios bacterianos más frecuentes: Implicaciones Clínicas. Med Clin. 2001 Apr; 116(14): 5419.

3. Adegbola R, DeAntonio R, Hill P, Roca A, Usuf E, Hoet B, Greenwood B. Carriage of Streptococcus pneumoniae and Other Respiratory Bacterial Pathogens in Low and Lower-Middle Income Countries: A Systematic Review and Meta-Analysis. PLoS ONE. 2014 Nov; 9(8): e103293. doi:10.1371/journal.pone.0103293.

4. World Health Organization. Estimated Hib and pneumococcal deaths for children under 5 years of age, 2008 [Internet]. Geneva: WHO. 2008 [updated 2013 Dec; citado el 12 de diciembre de 2015]. Disponible en: http://www.who.int/immunization/monitoring_surveillance/burden/estimates/Pneumo_ hib/en/index.html

5. Organización Mundial de la Salud/Organización Panamericana de la Salud (OMS/OPS). Indicadores Básicos. Situación de Salud en las Américas. [Internet]. Geneva: WHO. 2016 [updated 2013 Dec; citado el 12 de diciembre de 2015]. Disponible en: http://iris.paho.org/xmlui/handle/123456789/31288

6. Lynch JP \& Zhanel GG. Streptococcus pneumoniae: Epidemiology and risk factors, evolution of antimicrobial resistance, and impact of vaccines. Curr Opin Pulm Med. 2010 Dec; 16: 217-25.

7. Westerink MA, Schroeder Jr HW, Nahm MH. Immune responses to pneumococcal vaccines in children and adults: Rationale for age-specific vaccination. Aging Dis. 2012 Oct; 3:51-67.

8. Salleras L, Domínguez A, Izquierdo C. Epidemiología de la enfermedad neumocócica en el adulto. In: Moraga Llop FA, editor. La enfermedad neumocócica y su prevención. Caminando hacia el futuro. 1 ${ }^{\mathrm{a}}$. ed. Madrid; F. A. Moraga Llop; 2010. P. 126.

9. Feldman C. Pneumonia in the elderly. Clin Chest Med. 1999 Jul; 20: 563-73.

10. Obaro S, Adegbola R. The pneumococos: carriage, disease and conjugate vaccine. J. Med. Microbiol. 2002 Mar; 51: 98-104.

11. Van Hoek AJ, Andrews N, Waight PA, Stowe J, Gates P, George R, et al. The effect of underlying clinical conditions on the risk of developing invasive pneumococcal disease among hospitalized patients in England. J Infect. 2012 Apr; 65:17-24. 
Requena-Certad et al.

12. Leiberman A, Dagan R, Leibovitz E, Yagupsky P, Fliss D. The bacteriology of the nasopharynx in childhood. Int J Pediatr Otorhinolaryngol. 1999 Sept; 49(1): S151-3.

13. Lucarevschi BR, Baldacci ER, Bricks LF. Oropharyngeal carriage of Streptococcus pneumoniae by children attending day care center care in Tabauté SP: correlation between serotypes and the conjugated heptavalent pneumococcal vaccine. J. Pediatric (Rio J). 2003 Oct; 79 (3): 215-20.

14. Manrique M \& Cercado H. Neumonía adquirida en la comunidad en pacientes adultos hospitalizados en el HNDAC durante el 2003. Rev. Soc Peruana Neumol. 2005 Dec; 49(3): 23-31.

15. Simell B, Auranen K, Kayhty H, Goldblatt D, Dagan R, et al. The fundamental link between pneumococcal carriage and disease. Expert Rev Vaccines. 2012 Abr; 11: 841855.

16. Saha SK, Baqui AH, Darmstadt GL, Ruhulamin M, Hanif M, et al. Comparison of antibiotic resistance and serotype composition of carriage and invasive pneumococci among Bangladeshi children: implications for treatment policy and vaccine formulation. J Clin Microbiol. 2003 May; 41: 5582-5587.

17. Roca A, Hill PC, Townend J, Egere U, Antonio M, et al. Effects of community-wide vaccination with PCV-7 on pneumococcal nasopharyngeal carriage in the Gambia: a cluster-randomized trial. PLoS Med. 2011 Oct; 8: e1001107.

18. Abdullahi O, Nyiro J, Lewa P, Slack M, Scott JA. The descriptive epidemiology of Streptococcus pneumoniae and Haemophilus influenza nasopharyngeal carriage in children and adults in Kilifi district, Kenya. Pediatr Infect Dis. 2008 Jan; 27: 59-64.

19. Abdullahi O, Karani A, Tigoi CC, Mugo D, Kungu S, et al. Rates of acquisition and clearance of pneumococcal serotypes in the nasopharynges of children in Kilifi District, Kenya. J Infect Dis. 2012 Jun; 206: 1020-29.

20. Moyo SJ, Steinbakk M, Aboud S, Mkopi N, Kasubi M, et al. Penicillin resistance and serotype distribution of Streptococcus pneumoniae in nasopharyngeal carrier children under 5 years of age in Dar es Salaam, Tanzania. J Med Microbiol. 2012 Sep; 61: 952959.

21. Regev-Yochay G, Abullaish I, Malley R, Shainberg B, Varon M, et al. Streptococcus pneumoniae carriage in the Gaza strip. PLoS One. 2012 Jan; 7: e35061.

22. Denno DM, Frimpong E, Gregory M, Steele RW. Nasopharyngeal carriage and susceptibility patterns of Streptococcus pneumoniae in Kumasi, Ghana. West Afr J Med. 2002 May; 21: 233-6.

23. Rupa V, Isaac R, Manoharan A, Jalagandeeswaran R, Thenmozhi M. Risk factors for upper respiratory infection in the first year of life in a birth cohort. Int $\mathrm{J}$ Pediatr Otorhinolaryngol. 2012 Apr; 76: 1835-39.

24. Soewignjo S, Gessner BD, Sutanto A, Steinhoff M, Prijanto M, et al. Streptococcus pneumoniae nasopharyngeal carriage prevalence, serotype distribution, and resistance 
patterns among children on Lombok Island, Indonesia. Clin Infect Dis. 2001 Dec; 32: 1039-43.

25. Vu HT, Yoshida LM, Suzuki M, Nguyen HA, Nguyen CD, et al. Association between nasopharyngeal load of Streptococcus pneumoniae, viral coinfection, and radiologically confirmed pneumonia in Vietnamese children. Pediatr Infect Dis J. 2011 Jul; 30: 11-8.

26. Altuzarra R, Valenzuela T, Trucco O, Inostroza J, Granata P, Fleiderman J. Portación nasal de Streptococcus pneumoniae en adulto mayor y su respuesta frente a la vacunación antineumocócica. Rev Méd Chile. 2007 May; 135: 160-6.

27. Watt JP, O' Brien KL, Katz S, Bronsdon E, Dallas J et al. Nasopharyngeal versus Oropharyngeal. Sampling for Detection Pneumococcal Carriage in Adults. J Clin Microbiol. 2004 Jun; 42: 4974-6.

28. Quintero B \& Araque M. Perfil serológico y antibiotipia de cepas de Streptococcus pneumoniae aisladas de portadores nasales pediátricos. Invest Clín. 2006 Apr; 47(1): 17-26.

29. Castellano-González M, Perozo-Mena A, Ginestre-Pérez M, Avila-Roo Y, Romero-Añez $\mathrm{S}$, Harris-Socorro B, et al. Portadores nasales de Haemophilus influenzae en un Preescolar de Maracaibo. Bol Soc Ven Microbiol. 2000 Sept; 2(1):18-24.

30. Pezzlo M. Bacteriology aeróbica. In: Isenberg, H. (ed). Clinical Microbiology Procedures Handbook. $1^{\mathrm{a}}$ ed. Washington, DC: Editorial American Society for Microbiology: 1992. Cap 1: 1.1.1-1.1.30.

31. Paz C, Zoccoli C, Tobouti N, Sinto S. Cultura do trato Respiratorio Superior. In: Paz, C. (ed) Procedimentos Básicos en Microbiología Clínica. $1^{\text {a }}$ ed. Brasil: Sarvier Editora de Livros Médicos Ltda. 2000; Cap 15: 111-5.

32. Clinical and Laboratory Standards Institute (CLSI). Performance standards for antimicrobial susceptibility testing. Fourteenth Informational Supplement M100-S14; 2010.

33. WMA (World Medical Association). Declaración de Helsinki. Recommendations guinding physicians in biomedical research involving human subjects. JAMA. 1997 May; 277: 925-6.

34. WHONET. Sistema internacional de vigilancia de la resistencia. Organización Mundial de la Salud. [En Línea]. 2005. Disponible en: http: //www.Whonet.paho.org.

35. Edwards KM. Pneumococcal infections: therapeutics strategies and pitfalls. In: Tuomanen EI, Mitchell TJ, Morrison DA, Spratt BG. (editors). The pneumococcus: Washington: ASM Press American Society for Microbiology. 2004; Cap. 21: 314-30.

36. Mella S, Blamey R, Paiva O, Yáñez J, Riedel G, Aylwin M. et al. Impacto de la evaluación infectológica en el uso racional de antimicrobianos en pacientes con bacteriemia por Streptococcus pneumoniae. Rev Chil Infect. 2007 Apr; 24(4): 264-9. 
Requena-Certad et al.

37. Niederman M, Mandell L, Anzueto A, Bass J, Broughton W, Campbell G, et al. Guidelines for the management of adults with community-acquired pneumonia. Diagnosis, assessment of severity, antimicrobial therapy, and prevention. Am J Respir Crit Care Med. 2001 Jan; 163: 1730-54.

38. Mandell L, Bartlett J, Dowell S, File Jr T, Musher D, Whitney C. Update of practice guidelines for the management of community-acquired pneumonia in immunocompetent adults. Clin Infect Dis. 2003 Jul; 37: 1405-33.

39. Castellano M, Perozo A, Ginestre M, Avila Y. Portadores nasofaríngeos de bacterias potencialmente patógenas en pre-escolares de Maracaibo (2000-2001). Kasmera. 2002 Sept; 30(1):17-32.

40. Perozo-Mena A, Castellano M, Avila Y, Ginestre M, Fuenmayor A. Streptococcus pneumoniae: Estado de portador en niños preescolares y susceptibilidad a los antimicrobianos. XXXVII Jornadas Venezolanas de Microbiología. Trujillo. 4 al 6 de noviembre del 2001. p. 62. (Resúmenes).

41. Solórzano F, Ortiz L, Miranda M, Echaniz G, Soto A, Guiscafré H. Serotipos prevalentes de Streptococcus pneumoniae colonizadores de nasofaringe en niños del Distrito Federal. Sal Pub Mex. 2005 Abr; 47 (4): 276-1.

42. Zelaya, H. 2008. Frecuencia de portadores nasales de S. pneumoniae en niños menores de 6 años de la comunidad y de las Salas de Pediatría del Hospital Escuela Dr. Oscar Danilo Rosales Arguello. Tesis para optar al título de especialista en Pediatría. Hospital Escuela Dr. Oscar Danilo Rosales Arguello. Facultad de ciencias médicas. Universidad Nacional Autónoma De Nicaragua Unan-León. P. 46 (Multígrafo).

43. Raman AS, Swinburne AJ, Fedullo AJ. Pneumococcal adherence to the buccal epithelial cells of cigarette smokers. 1983 Jul; Chest. 83: 23-7.

44. Dye JA, Adler KB. Effects of cigarette smoke on epithelial cells of the respiratory tract. Thorax. 1994. May; 49: 825-34.

45. Mili F, Flanders WD, Boring JR, Annest JL, Destefano F. The associations of race, cigarette smoking, and smoking cessation to measures of the immune system in middleaged men. Clin Immunol Immunopathol. 1991 Sep; 59: 187-200.

46. Sankilampi U, Isoaho R, Bloigu A, Kivela S1, Leinonen M. Effect of age, sex and smoking habits on pneumococcal antibodies in an elderly population. Int J Epidemiol. 1997 Dec; 26: 420-7.

47. Nuorti JP, Butler JC, Farley MM, Harrison LH, Mcgeer A, Kolczak MS, et al. Cigarette smoking and invasive pneumococcal disease. N Engl J Med. 2000 Apr; 342: 681-9.

48. Moore M, Qazis S, Shindo N. Neumonía neumocócica. En: Heymann DL, editor. El control de las enfermedades transmisibles. 19a ed. Washington, D. C.: American Public Health Association-OPS. 2011. P. 560.

49. Gosalbes-Soler V, Márquez-Calderón S, Maiques-Galán A, Latour Pérez J, BernalDelgado E, Puig-Barberà J, et al. Actividades preventivas en atención primaria: 
identificación de áreas de concordancia entre guías de práctica clínica basadas en la evidencia. Med Clin (Barc). 2000 Jul; 114(Supl 2):88-92.

50. OMS. Vacuna conjugada neumocócica. Boletín Epidemiológico Semanal 2007. 2007 Jun; 82(12): 93-04.

51. Austrian R. Surveillance of pneumococcal infection for field trials of polyvalent pneumococcal vaccines. DAB-VDP-12-84, 1-84. Bethesda (MD), National Institute of Health. 1980. P. 85.

52. Fedson D \& Liss C. Precise answers to the wrong question: prospective clinical trials and the meta-analyses of pneumococcal vaccine in elderly and high-risk adults. Vaccine. 2004 May; 22: 927-946.

53. Casas Maldonado F, Alfageme Michavila I, Barchilón Cohen VS, Peis Redondo JI, Vargas Ortega DA. Recomendación de la vacuna antineumocócica en las enfermedades respiratorias crónicas. Semergen. 2013 Nov; 40(6): 313-25.

54. Christenson B \& Lundbergh P. Comparison between cohorts vaccinated and unvaccinated against influenza and pneumococcal infection. Epidemiol Infect. 2002 Jan; 129: 515-24.

55. Casellas JM \& Cha Torea JC. 2000. Guía de terapéutica antimicrobiana. En: Terapia Intensiva. Buenos Aires, Argentina: Wyeth-Ayerst Arg. P. 121.

56. Gómez M. Resistencia del neumococo a los antibióticos. Experiencia en Venezuela. Bol Soc Venez Microbiol. 1986 Oct; 6:2-4.

57. Gómez M, Galindo D, Medina G, Cedeño M, Priscelli A. Sensibilidad de Streptococcus pneumoniae a la penicilina y cefalosporinas. Bol Soc Ven Microbiol. 1998 Oct; 19(1): 17-20.

58. Grupo Venezolano De Vigilancia De La Resistencia Bacteriana. Actualización de los datos de resistencia bacteriana a los antimicrobianos en Venezuela. Período julio 2001diciembre 2002. Rev Soc Ven Microbiol. 2003 Oct; 23(1):89-97.

59. Zamora L. 2004. Susceptibilidad del Streptococcus pneumoniae a la penicilina en Neumonía adquirida en la comunidad Complejo Hospitalario Universitario "Ruiz y Páez" Ciudad Bolívar - Estado Bolívar, noviembre 2002-noviembre 2003. Tesis de Post-Grado. Dpto de Medicina Interna. Complejo Hospitalario Universitario Ruíz y Páez. Esc. Cs. Salud. Bolívar. U.D.O. P 81.

60. Programa Venezolano De Vigilancia De Resistencia Bacteriana A Los Antimicrobianos En Venezuela. 2011. Disponible en: http://www.provenra.org/programa.html

61. Casellas JM. Resistencia a los antibacterianos en América Latina: consecuencias para la infectología. Rev Panam Salud Pública. 2011 Sep; 30(6):519-28.

62. Cardoso G \& Velázquez N. Importancia clínica del Streptococcus pneumoniae tolerante a la Vancomicina. Enf Infec Ped. 2007 Jun; 20(79): 51-3. 
Requena-Certad et al.

63. Mbelle N, Huebner R, Wasas A, Kimura A, Chang I, Klugman K. Immunogenicity and impact on nasopharyngeal carriage of nonvalent pneumococcal conjugate vaccine. $\mathrm{J}$ Infect Dis. 1999 Feb; 180:1171-6. 
Figuras

$$
\begin{gathered}
\text { CUADRO } 1 \\
\text { PACIENTES PEDIÁTRICOS PORTADORES NASOFARÍNGEOS Y NO } \\
\text { PORTADORES DE Streptococcus pneumoniae SEGÚN EDAD. } \\
\text { CIUDAD BOLÍVAR. ESTADO BOLÍVAR. } \\
\text { ENERO 2009- DICIEMBRE } 2010 .
\end{gathered}
$$

\begin{tabular}{|c|c|c|c|c|c|c|}
\hline \multirow{3}{*}{$\begin{array}{c}\text { Grupos de edad } \\
\text { (años) }\end{array}$} & \multicolumn{4}{|c|}{ Portadores Nasofaríngeos } & \multirow{2}{*}{\multicolumn{2}{|c|}{ Total }} \\
\hline & \multicolumn{2}{|c|}{$\mathbf{S i}$} & \multicolumn{2}{|c|}{ No } & & \\
\hline & $\mathbf{N}^{\mathbf{0}}$ & $\%$ & $\mathbf{N}^{\mathbf{0}}$ & $\%$ & $\mathbf{N}^{0}$ & $\%$ \\
\hline Lactante Menor & 0 & 0,00 & 17 & 21,25 & 17 & 21,25 \\
\hline Lactante Mayor & 1 & 1,25 & 11 & 13,75 & 12 & 15,00 \\
\hline Preescolar & 1 & 1,25 & 9 & 48,75 & 40 & 50,00 \\
\hline Escolar & 1 & 1,25 & 10 & 12,50 & 11 & 13,75 \\
\hline Total & 3 & 3,75 & 77 & 96,25 & 80 & 100,00 \\
\hline
\end{tabular}


Requena-Certad et al.

\section{CUADRO 2}

INDIVIDUOS DE LA TERCERA EDAD INSTITUCIONALIZADOS PORTADORES

FARÍNGEOS Y NO PORTADORES DE Streptococcus pneumoniae SEGÚN EDAD.

CIUDAD BOLÍVAR. ESTADO BOLÍVAR.

ENERO 2011- DICIEMBRE 2011.

\begin{tabular}{|c|c|c|c|c|c|c|}
\hline \multirow{3}{*}{$\begin{array}{c}\text { Grupos de edad } \\
\text { (años) }\end{array}$} & \multicolumn{4}{|c|}{ Portadores faríngeos } & \multicolumn{2}{|c|}{ Total } \\
\hline & \multicolumn{2}{|c|}{ Si } & \multicolumn{2}{|c|}{ No } & & \\
\hline & $\mathbf{N}^{\mathbf{o}}$ & $\%$ & $\mathbf{N}^{\mathbf{0}}$ & $\%$ & $\mathbf{N}^{\mathbf{0}}$ & $\%$ \\
\hline 51-60 & 1 & 1,52 & 4 & 6,06 & 5 & 7,58 \\
\hline $61-70$ & 2 & 3,03 & 13 & 19,70 & 15 & 22,73 \\
\hline $71-80$ & 4 & 6,06 & 16 & 24,24 & 20 & 30,30 \\
\hline $81-90$ & 3 & 4,55 & 16 & 24,24 & 19 & 28,79 \\
\hline$>90$ & 1 & 1,52 & 6 & 9,09 & 7 & 10,61 \\
\hline Total & 10 & 15,15 & 51 & 77,27 & 66 & 100,00 \\
\hline
\end{tabular}




\section{CUADRO 3}

PACIENTES PEDIÁTRICOS PORTADORES FARÍNGEOS DE Streptococcus pneumoniae SEGÚN ANTECEDENTES PERSONALES Y PSICOBIOLÓGICOS.

CIUDAD BOLÍVAR. ESTADO BOLÍVAR.

ENERO 2009- DICIEMBRE 2010.

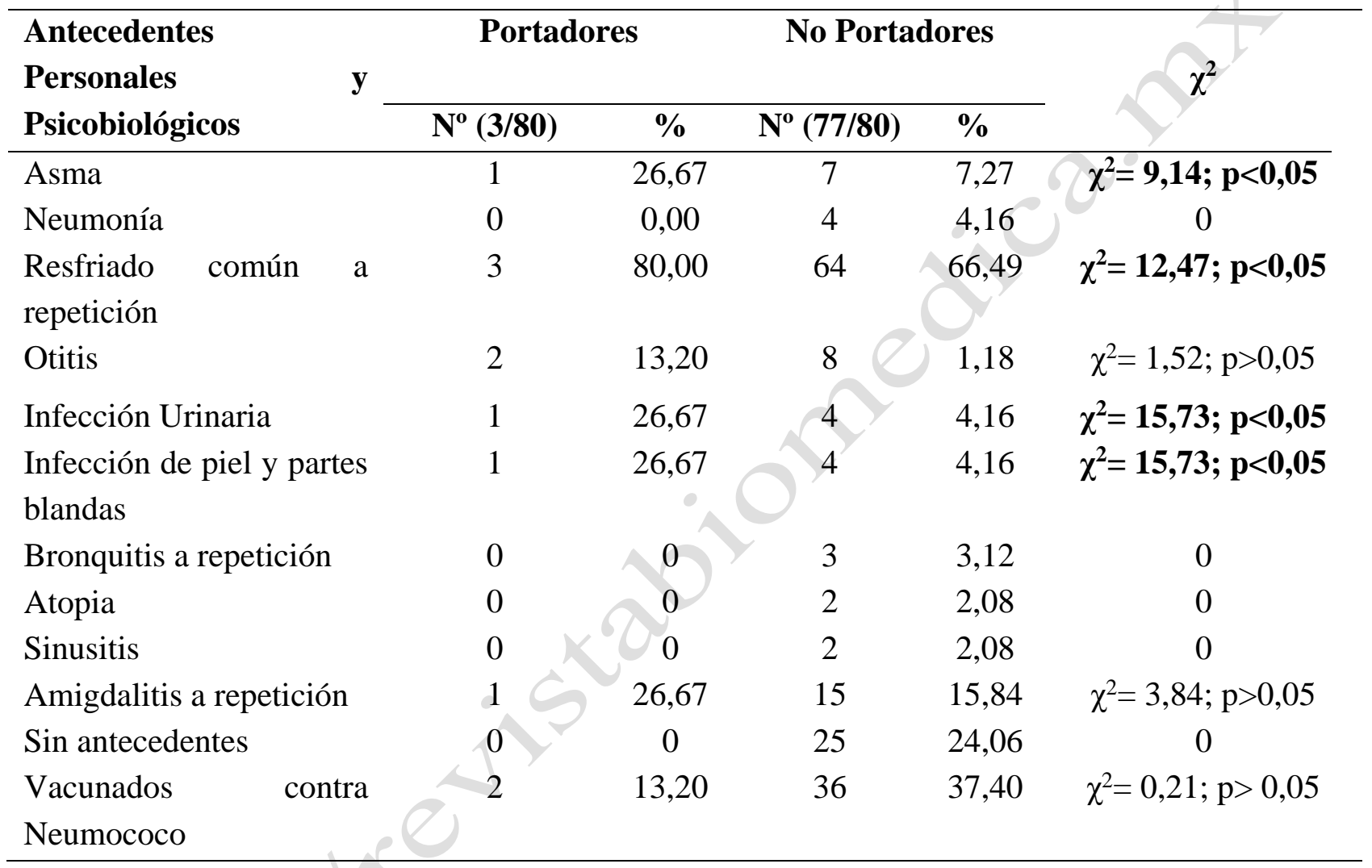


Requena-Certad et al.

CUADRO 4

INDIVIDUOS DE LA TERCERA EDAD INSTITUCIONALIZADOS PORTADORES

FARÍNGEOS DE Streptococcus pneumoniae SEGÚN ANTECEDENTES PERSONALES

Y PSICOBIOLÓGICOS. CIUDAD BOLÍVAR. ESTADO BOLÍVAR.

ENERO 2011- DICIEMBRE 2011.

\begin{tabular}{|c|c|c|c|c|c|}
\hline \multirow{3}{*}{$\begin{array}{l}\text { Antecedentes } \\
\text { Personales } \\
\text { Psicobiológicos }\end{array}$} & \multicolumn{2}{|c|}{ Portadores } & \multicolumn{2}{|c|}{ No Portadores } & \multirow[t]{3}{*}{+4} \\
\hline & & & & & \\
\hline & $N^{\circ}(10 / 66)$ & $\%$ & $N^{\circ}(56 / 66)$ & $\%$ & \\
\hline Hipertensión arterial & 8 & 52,8 & 11 & 12,96 & $\chi^{2}=0,12 ; p>0,05$ \\
\hline Cardiopatía Hipertensiva & 6 & 39,6 & 8 & 9,43 & $\chi^{2}=3,77 ; p>0,05$ \\
\hline Diabetes Mellitus Tipo II & 4 & 26,4 & 15 & 17,68 & $\chi^{2}=4,31 ; p<0,05$ \\
\hline $\begin{array}{l}\text { Enfermedad pulmonar } \\
\text { obstructiva crónica }\end{array}$ & 2 & 13,2 & 1 & 1,18 & 0 \\
\hline Incontinencia urinaria & 3 & 19,8 & 1 & 1,18 & 0 \\
\hline $\begin{array}{l}\text { Enfermedad cerebro- } \\
\text { vascular }\end{array}$ & 1 & 6,6 & 5 & 5,89 & 0 \\
\hline Antecedentes Tabáquicos & 7 & 46,2 & 14 & 16,5 & $\chi^{2}=0,11 ; p>0,05$ \\
\hline Vacunados & 4 & 26,4 & 6 & 7,07 & $\chi^{2}=13,85 ; p<0,05$ \\
\hline Neumococo & & & & & \\
\hline
\end{tabular}

